\title{
Aprendizaje basado en problemas: evaluación de una propuesta curricular para la formación inicial docente
}

\author{
Problem Based Learning: assessment of a PBL curricular proposal for \\ pre-service teacher training
}

\section{Aprendizagem baseada em problemas: avaliação de uma proposta curricular para a formação docente inicial}

\author{
Óscar Paineán Bustamante ${ }^{1}$, Verónica Aliaga Prieto ${ }^{2}$, \\ Teresa Torres Torres ${ }^{3}$ \\ ${ }^{1}$ Universidad de Atacama, oscar.painean@uda.cl \\ ${ }^{2}$ Universidad de Atacama, veronica.aliaga@uda.cl \\ ${ }^{3}$ Universidad de Atacama, teresa.torres@uda.cl
}

\begin{abstract}
RESUMEN
Las distintas miradas y lecturas que hacemos de los avances en psicología y educación nos llevan a preguntarnos cómo mejorar el aprendizaje en nuestros estudiantes y cuál es la mejor forma de orientarlos a descubrir el conocimiento. Sabemos que la tendencia es dejar de ser, nosotros, los profesores, los protagonistas y dejar que lo sean los estudiantes. En el trabajo pedagógico, a nivel universitario, existe una oportunidad muy clara para implementar alternativas metodológicas innovadoras que provoquen un mayor éxito en el aprendizaje de los alumnos. En el presente artículo se describe una experiencia metodológica basada en el aprendizaje activo y que coloca al alumno en el centro del proceso de enseñanza. Se muestran los resultados observados al implementar el enfoque curricular de aprendizaje basado en problemas (ABP) en la Facultad de Humanidades y Educación de la Universidad de Atacama.
\end{abstract}

Palabras clave: aprendizaje basado en problemas, enfoque curricular, estudiantes.

\begin{abstract}
The different looks and readings that we do of the progress in current psychology and education make us reflect on how to improve our students' learning and what is the best way to help them discover knowledge by themselves. We know that the new trend is for teachers to stop being the protagonists, allowing the students to take up this role. When teaching at the university level, there is a good chance of implementing innovative methodological alternatives to promote great success in students' learning. The present article describes a methodological experience on active learning -known as problem -based learning (PBL) which places the student in the center of the learning process. The experience relates to students from the School of Humanities and Education of the University of Atacama. The results are shown in the present article.
\end{abstract}

Key words: problem-based learning, curricular approach, students.

\section{RESUMO}

Os diferentes olhares e leituras feitas dos avanços relacionados à psicologia e educação, levam a questionamentos sobre como melhorar a aprendizagem de estudantes e sobre qual é a melhor forma de orientá-los para descobrirem o conhecimento. Reconhece-se a tendência de professores deixarem de ser protagonistas a fim de que estudantes passem a sê-los. No trabalho pedagógico em nível universitário, há oportunidade bastante clara para implementar alternativas metodológicas inovadoras por meio das quais estudantes obtenham mais êxito na aprendizagem. Descreve-se uma experiência metodológica baseada na aprendizagem ativa, na qual o estudante é centro do processo de ensino. 
Mostram-se os resultados observados ao implementar o enfoque curricular de Aprendizagem Baseada em Problemas (ABP), na Faculdade de Humanas e Educaçâo da Universidade de Atacama.

Palavras chave: aprendizagem baseada em problemas, enfoque curricular, estudantes.

\section{INTRODUCCIÓN}

Este trabajo resume los resultados de una investigación ${ }^{1}$ destinada a conocer cómo los estudiantes de las carreras pedagógicas de la Facultad de Humanidades y Educación, de la Universidad de Atacama, evalúan el impacto en su formación profesional del actual currículo de formación inicial docente que se implementara, en esta Facultad, a partir del año 2000, como resultado del proyecto FFID "Docentes para el Siglo XXI. Reforma Integral del Currículo". ${ }^{2}$

Este diseño curricular, definido como "Híbrido Secuencial" (Juan Iglesias, 2002; Pamela Labra et al., 2007), incorpora al diseño curricular clásico, por asignaturas, un nuevo componente: módulos de "Aprendizaje Basado en Problemas" (ABP), los que se encadenan en un desarrollo secuencial de módulos disciplinarios con módulos de ABP.

La metodología ABP fue desarrollada y llevada a la práctica en los años 60's en la Escuela de Medicina de la Universidad de Mc Master, Canadá. Desde entonces, ha habido un gran impulso de este enfoque educativo en la educación superior, convirtiéndose en un componente fundamental de muchos currículos de formación profesional.

Dentro de las universidades que han implementado esta metodología en sus currículos de formación se pueden nombrar a las siguientes: Wheeling Jesuit University, WV, U.S.A.; Universidad de Maastricht, Holanda; Instituto Tecnológico de Monterrey, México; New Castle, Australia.

Es importante destacar que la metodología ABP ha sido aplicada preferentemente en las Facultades de Medicina, Ciencias de la Salud, Leyes, Economía, Administración y Ciencias Culturales. En Educación, una de las primeras experiencias chilenas se desarrolla en la Universidad de Atacama a partir del año 2000, como se ha señalado anteriormente.

Luego de ocho años de aplicación del "Currículo Híbrido-Secuencial" de la metodología $\mathrm{ABP}$ en la formación inicial de todos los estudiantes de pedagogía de esta universidad, y de haber egresado cuatro cohortes de estudiantes, se hizo necesario comenzar una evaluación de este proceso, desde la perspectiva de los profesores en formación. Para este efecto, nos propusimos evaluar tres aspectos de su aplicación: 1) el modo cómo evalúan los alumnos el impacto de este nuevo modelo curricular en su formación y, en especial, el de los módulos ABP, a través de cuestionarios de respuesta abierta. 2) cómo el modelo ABP ha cumplido con los objetivos declarados en la justificación de su aplicación y 3) cómo se ha implementado el sistema desde un punto de vista operativo, por ejemplo: organización, desempeño de tutores, tiempos de desarrollo, integración con los otros componentes del diseño curricular, etc.

\footnotetext{
1 Proyecto: "El Aprendizaje Basado en Problemas: Cómo lo Evalúan los Estudiantes de la Facultad de Humanidades y Educación de la Universidad de Atacama” Investigador Responsable: Óscar Paineán Bustamante; Coinvestigadoras: Verónica Aliaga Prieto y Teresa Torres Torres. Financiado por la Dirección de Investigación y Postgrado de la Universidad de Atacama, año 2007.

2 Proyecto “Docentes para el Siglo XXI: Reforma Integral del Currículo”, Copiapó, Universidad de Atacama, 2000 .
} 


\section{CURRÍCULO HÍBRIDO-SECUENCIAL (C.H-S): UNA NUEVA PROPUESTA ${ }^{3}$}

En el marco del Proyecto FFID "Docentes para el Siglo XXI-Reforma Integral del Currículo", la Facultad de Humanidades y Educación de la Universidad de Atacama inició, en 1998, una profunda evaluación de sus procesos de formación inicial de Docentes, obteniendo como resultado la urgente necesidad de una alternativa curricular más moderna, que superara los déficit del currículo anterior, centrado en la enseñanza de contenidos, a veces de poca relevancia y pertinencia; desconectado de la realidad; apoyado en metodologías estereotipadas y poco significativas; basado exclusivamente en clases frontales; de nula integración y consistencia con el perfil profesional declarado para nuestros egresados, entre otras precariedades.

Se buscó, en consecuencia, una alternativa de formación docente que propendiera a formar un profesional reflexivo; con habilidades de aprendizaje independiente; con destrezas para la comunicación interpersonal; capaz de resolver problemas; capaz de trabajar en equipo; capaz de aplicar métodos de investigación científica y con destrezas para procesar información. Todas estas habilidades, declaradas en el perfil del nuevo profesional de la educación que se esperaba formar, deberían alcanzarse a través de este nuevo currículo y, en particular, como efecto de la aplicación del Aprendizaje Basado en Problemas.

\section{APRENDIZAJE BASADO EN PROBLEMAS}

En una brevísima caracterización del ABP. podríamos decir que se trata de una modalidad curricular y, al mismo tiempo, una metodología de enseñanza - aprendizaje con evidente base constructivista, que busca, a través del trabajo de equipo de un grupo pequeño de estudiantes (entre 8 y 10), orientados por un profesor - tutor, abordar un problema relacionado con su futuro ámbito profesional, recogido de la realidad, para luego de un proceso de investigación proponer soluciones, con la finalidad de que durante ese proceso cada uno de los alumnos participantes pueda construir un sinnúmero de aprendizajes altamente significativos, pertinentes, actualizados y contextualizados. Tan importante como la solución del problema es el proceso que implica el desarrollar todas las habilidades que ya hemos reseñado.

Lo importante acá es que el contexto de aprendizaje es la realidad misma: en efecto, junto con la investigación académico-bibliográfica, el equipo debe realizar investigación de campo (entrevistar, encuestar, etc.), lo que significa salir del aula y conocer la realidad en que se sitúan los problemas investigados.

Por otra parte, en esta metodología de enseñanza-aprendizaje, si bien el protagonista es el estudiante, no es menos importante la función del profesor-tutor. Su actuación es clave en este proceso: debe adecuarse a las condiciones del grupo, orientar la investigación, estimular el pensamiento del equipo a través de preguntas que iluminen las vías de acceso. El profesor acá debe olvidar su función de ser fuente de información para transformarse en un mediador entre la información disponible, el conocimiento de los alumnos y la naturaleza del problema que se aborda.

3 Una excelente descripción del C.H-S y del A.B.P. está en el artículo de Pamela Labra et al. (2007). 


\section{EVALUACIÓN DEL ABP}

La investigación que reseñamos en este trabajo, sobre cómo nuestros estudiantes evalúan el proceso de $\mathrm{ABP}$, es la primera que se ha realizado al interior de nuestra Facultad. Sin embargo, la aplicación de esta metodología de enseñanza-aprendizaje ha estado siendo permanentemente evaluada durante todo su desarrollo a través de jornadas de reflexión y análisis, al término de cada uno de los módulos desarrollados. En estas jornadas participan los mismos estudiantes junto con los profesores tutores.

Los principales problemas detectados en relación con la implementación del sistema ABP, cuestión que también está planteada en el objetivo $\mathrm{N}^{\circ} 3$ del Proyecto de Investigación, son, de modo sumario, los siguientes:

- Deficiente desempeño de algunos tutores

- Falta de compromiso de algunos tutores y alumnos

- Deficientes instrumentos para evaluar el desempeño de los estudiantes en los equipos.

- Problemas de coordinación interna y externa

- Mala formulación de los problemas presentados a los equipos

A pesar de estos problemas y de otros tal vez no menos importantes, existe una percepción de la mayoría de los tutores de que estos problemas se han ido superando paulatinamente y que cada vez las evaluaciones globales del proceso, que se realizan al final de cada módulo de ABP, han ido siendo cada vez más positivas.

\section{PRESENTACIÓN DE LA INVESTIGACIÓN}

\section{Objetivo General:}

Describir la evaluación que realizan los estudiantes de pedagogía de la Facultad de Humanidades y Educación de las cohortes 2002, 2003, 2004 y 2005, al término de sus módulos de Aprendizaje Basado en Problemas

\section{Objetivos Específicos:}

1. Completar información de la metodología ABP implementada en la Facultad de Humanidades y Educación, respecto de su desarrollo y organización, a través de la evaluación escrita de una muestra de 70 estudiantes, representantes de todas carreras de pedagogía, que corresponden a las cohortes 2002, 2003, 2004 y 2005 que han finalizado todos sus módulos.

2. Determinar si el desarrollo del proceso de los Módulos de ABP, al interior de la Facultad de Humanidades y Educación, es concordante con los objetivos base de la metodología del Aprendizaje Basado en Problemas.

3. Establecer si la planificación, organización e implementación realizada por la Facultad para el desarrollo de los módulos ABP son adecuadas, de acuerdo con la opinión de los estudiantes (tiempo de duración, tiempo de dedicación, formas de exposición, etc.). 


\section{Procedimiento:}

En esta investigación se trabajó con encuestas, estrategia de investigación basada en declaraciones verbales de una población concreta; en este caso, estudiantes de pedagogía de la Universidad de Atacama.

Para realizar la investigación mediante encuesta se recurrió a su instrumento básico para la recogida de información: el cuestionario, que consistió en un listado de preguntas estandarizadas, breves y fáciles de comprender por las personas a las que van dirigidas. A su vez, todas las preguntas presentadas en el cuestionario son de tipo preguntas abiertas, en las cuales el estudiante expresa, con sus propias palabras, las fortalezas y debilidades del proceso de ABP.

Los principales aspectos que se tuvieron en cuenta en el cuestionario y que corresponden a los que se trabajan en los módulos de Aprendizaje basados en problemas son los siguientes:

- Conocimiento: Se les consulta acerca de qué saben ellos del ABP; cuál es el concepto que ellos manejan y cuáles son los principales objetivos que tiene esta metodología

- Habilidades desarrolladas a través del ABP: Se les pregunta por las habilidades que permite desarrollar el ABP y, en este sentido, si consideran que este modelo aporta o no para su logro.

- Gradación: Aquí se consulta si han percibido algún tipo de gradación en el desarrollo del proceso, considerando que ellos han completado su ciclo ABP.

- Tutores: Aquí se les pide dos cosas: que evalúen el desempeño de los tutores que tuvieron durante todo el proceso y que describan las características ideales del tutor en los distintos niveles del proceso.

- Sugerencias: Se les solicitan sugerencias referidas a aspectos muy específicos de la implementación, por ejemplo la duración de los módulos, desempeño de la Comisión ABP encargada de los aspectos operativos del sistema, problemas, etc.

La población consultada corresponde a estudiantes de las cuatro carreras de pedagogías que tiene esta universidad: Pedagogía Básica, Pedagogía en Educación Parvularia, en Educación Física e Inglés. De este universo se estableció una muestra no probabilística de 70 alumnos, pertenecientes a las cohortes 2002, 2003, 2004 y 2005, que han finalizado la totalidad de sus módulos de ABP.

\section{RESULTADOS DE LA INVESTIGACIÓN}

Los resultados que se presentan corresponden a dos aplicaciones del cuestionario distanciadas por dos años. Los datos obtenidos se llevan a porcentaje y se presentan en forma de gráfico para facilitar su apreciación.

En la primera oportunidad, año 2005, se aplica el cuestionario a una muestra de treinta alumnos, representantes de todas las carreras pedagógicas de las cohortes de ingreso 2002-2003. En la segunda oportunidad, año 2007, se aplica el mismo 
cuestionario a una muestra de cuarenta estudiantes correspondientes a las cohortes de ingreso 2004-2005.

\section{Cuadro Comparativo:}

Tomando en cuenta que el instrumento de recolección de datos fue aplicado en dos oportunidades para observar cómo se comportan ambas muestras, se presentan los resultados de ambas aplicaciones:

\subsection{Considerando que usted ha terminado los módulos $A B P$, ¿Qué es para usted el ABP?}

$1^{\circ}$ aplicación

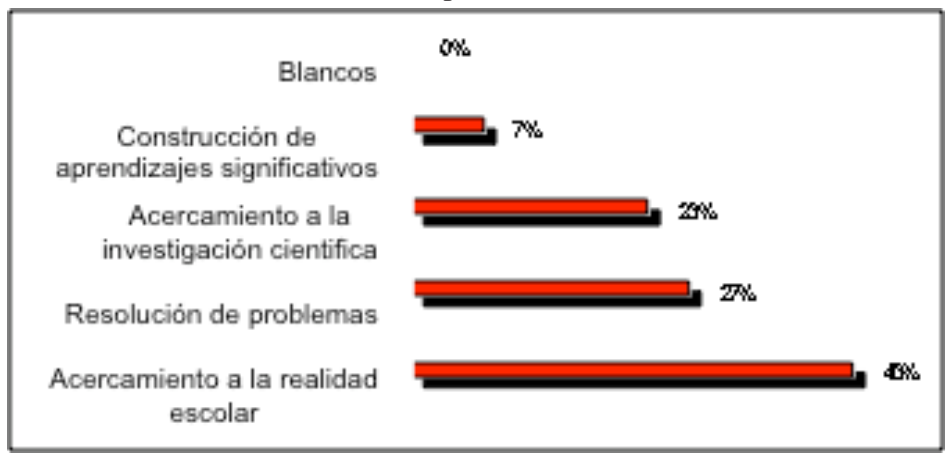

$2^{\circ}$ aplicación

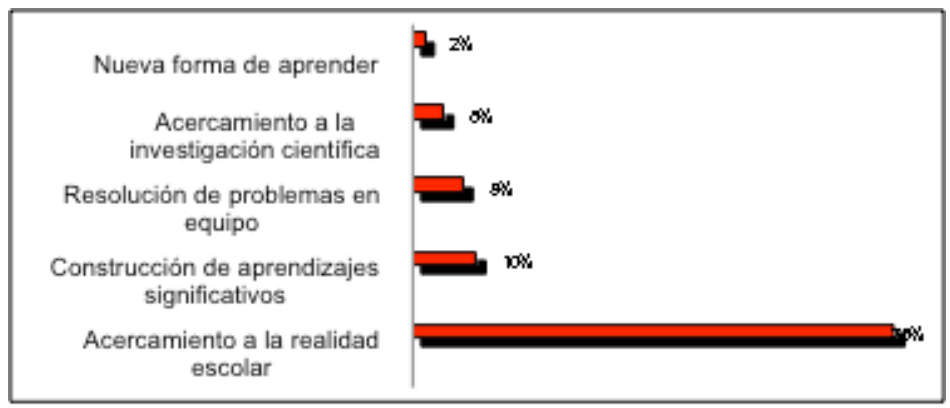

Como se puede observar en las dos aplicaciones del cuestionario, los alumnos de ambas muestras, que ya han terminado los módulos de Aprendizaje Basado en Problemas, los consideran en su mayoría como un acercamiento a la realidad escolar. 


\subsection{Según usted ¿Cuáles son los objetivos del ABP?}

$1^{\circ}$ aplicación

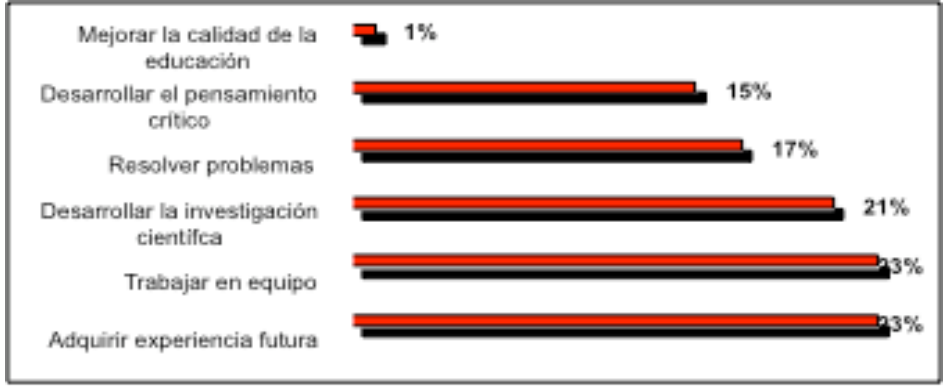

$2^{\circ}$ aplicación

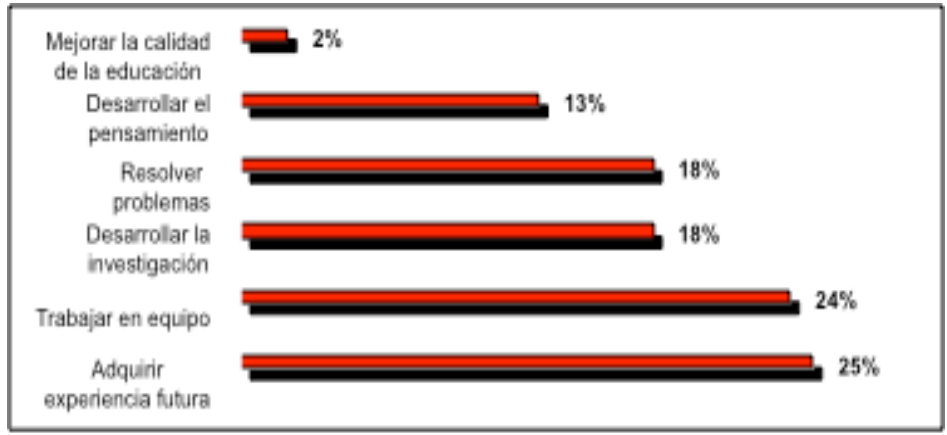

Las respuestas en ambas aplicaciones destacan la adquisición de experiencia futura y el trabajo en equipo.

\subsection{Nombre cuatro habilidades que usted considera importante y} que cree las aprendió por medio de los módulos ABP

$1^{\circ}$ aplicación

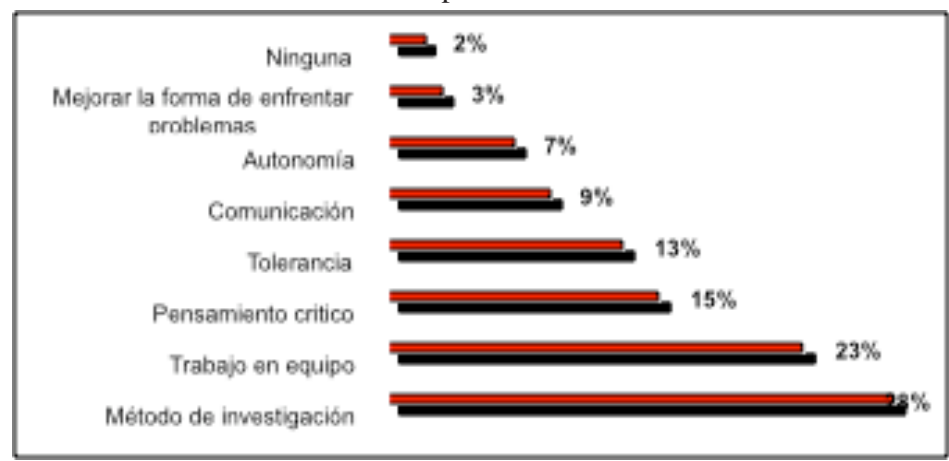


$2^{\circ}$ aplicación

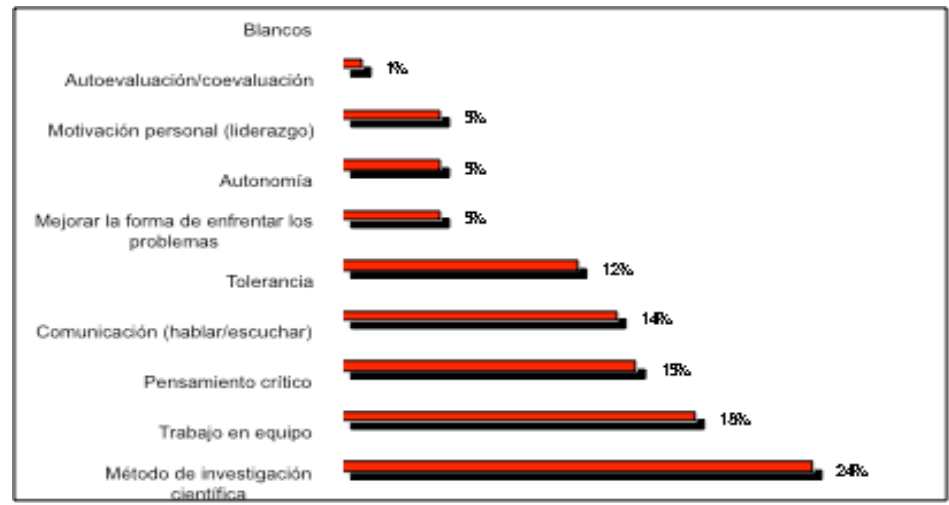

Los porcentajes mayores de respuesta se refieren al uso del método de investigación científica y al trabajo en equipo.

\subsection{Para su formación como profesional docente:}

¿Usted cree que los módulos ABP son un aporte? SI/NO, ¿Por qué?

\section{$1^{\circ}$ aplicación}

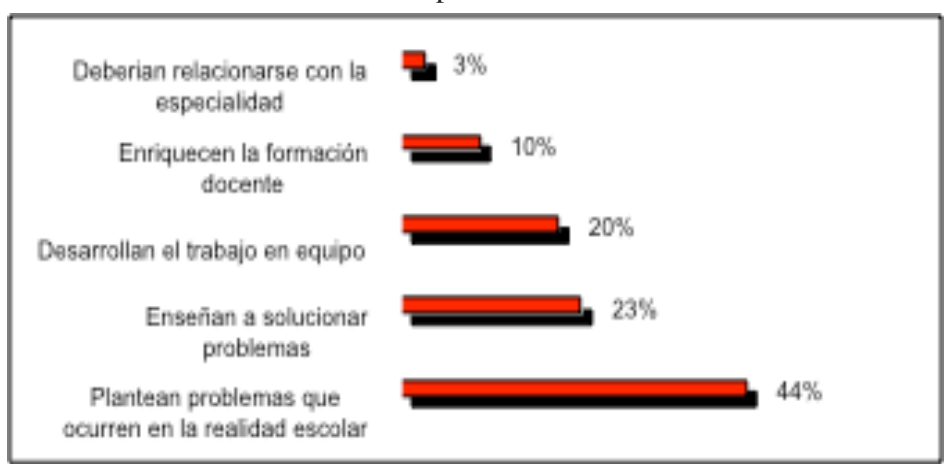

$2^{\circ}$ aplicación

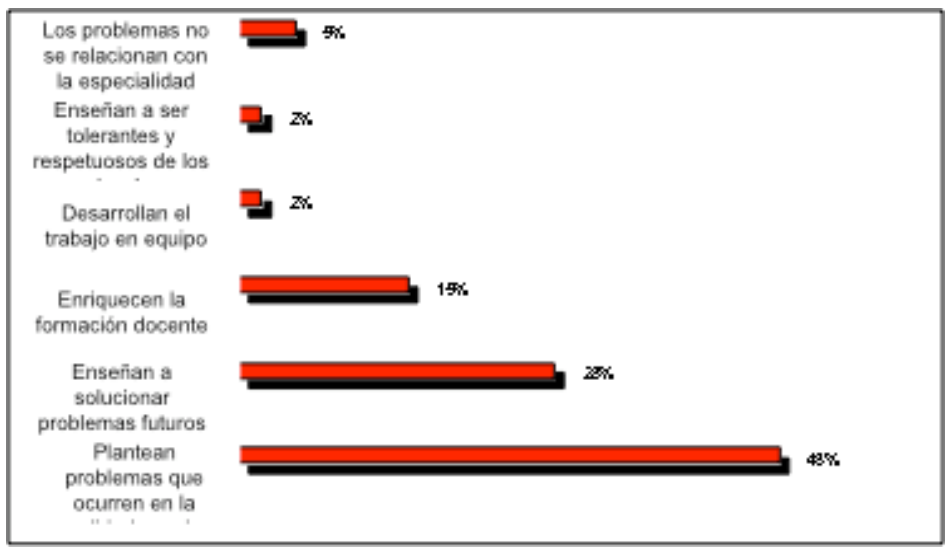


Gracias a los resultados entregados por los encuestados, podemos darnos cuenta que los ABP son considerados, por parte del alumnado, como un aporte para su formación profesional porque permiten resolver problemas que ocurren en la realidad escolar.

4.1. Durante el proceso de ABP, ¿Ustedes han percibido una secuencia de lo que se aprende en el nivel 100, 200 y 300? SI/NO. Si responde SÍ, expréselo a continuación.

Si responde NO, continúe con la pregunta 4.2.

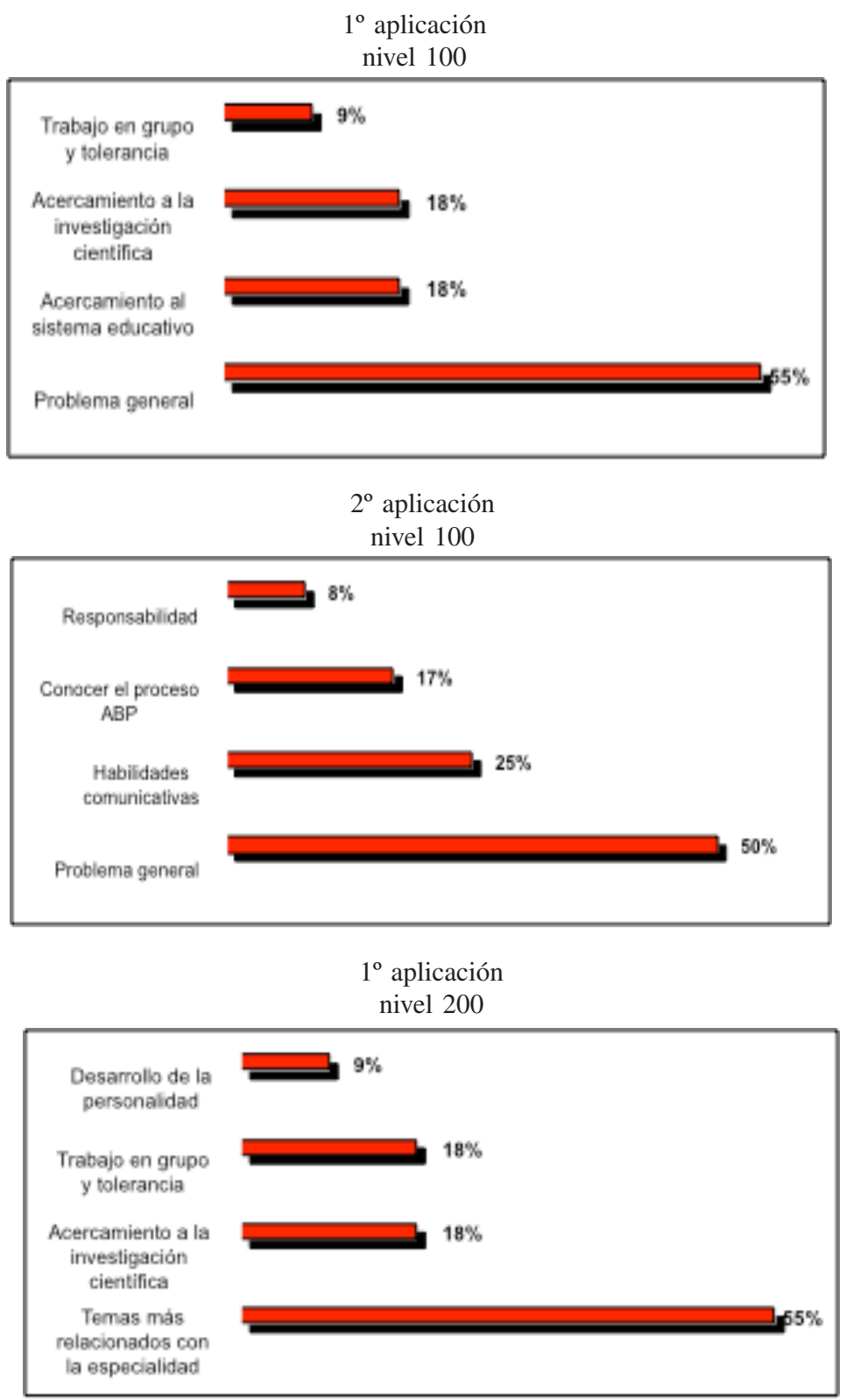


$2^{\circ}$ aplicación

nivel 200

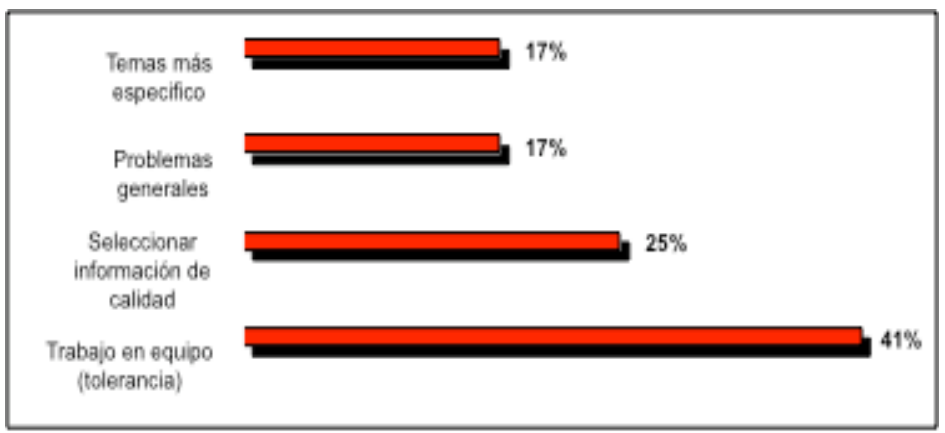

$1^{\circ}$ aplicación

nivel 300

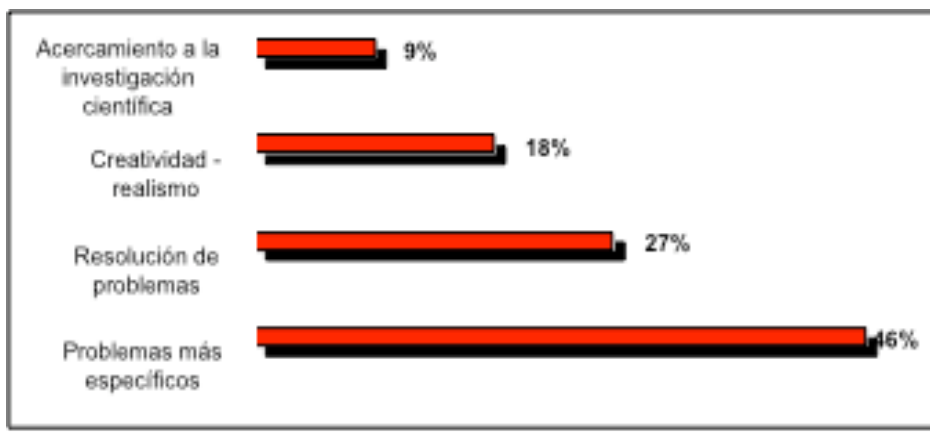

$2^{\circ}$ aplicación

nivel 300

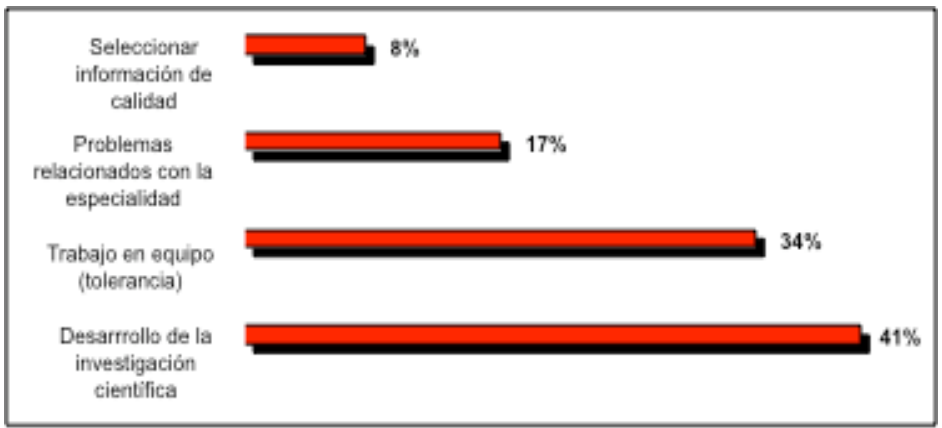

Cabe destacar que en esta pregunta las respuestas entregadas son muy variadas, pero, en general, los alumnos sí perciben una gradación de aprendizaje en los distintos módulos ABP que enfrentan durante su carrera. 
4.2. Si para usted existe otro tipo de gradación, explíquela a continuación. Asimismo, si usted siente que no ha habido ninguna gradación, plantéelo a continuación justificando su respuesta.

$1^{\text {o }}$ aplicación

no hay gradación

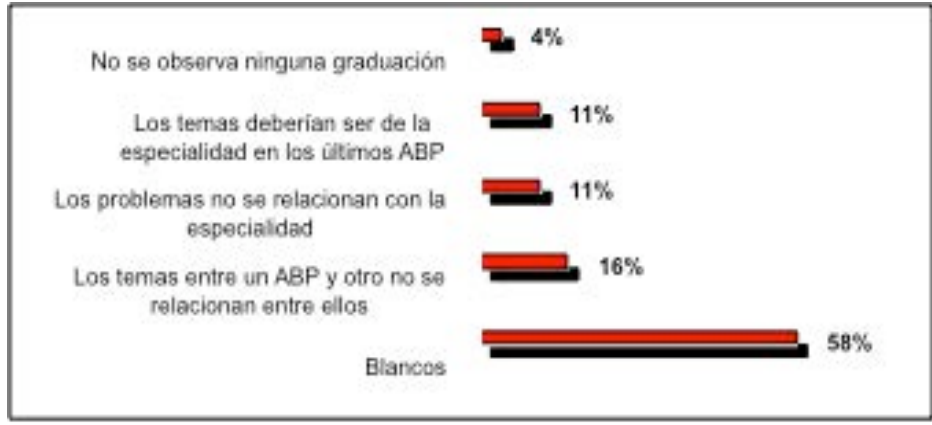

$2^{\circ}$ aplicación

no hay gradación

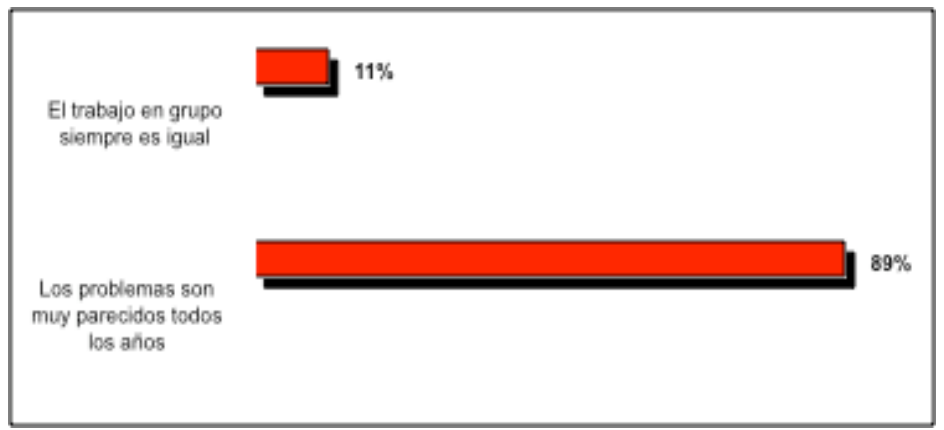

Otra gradación

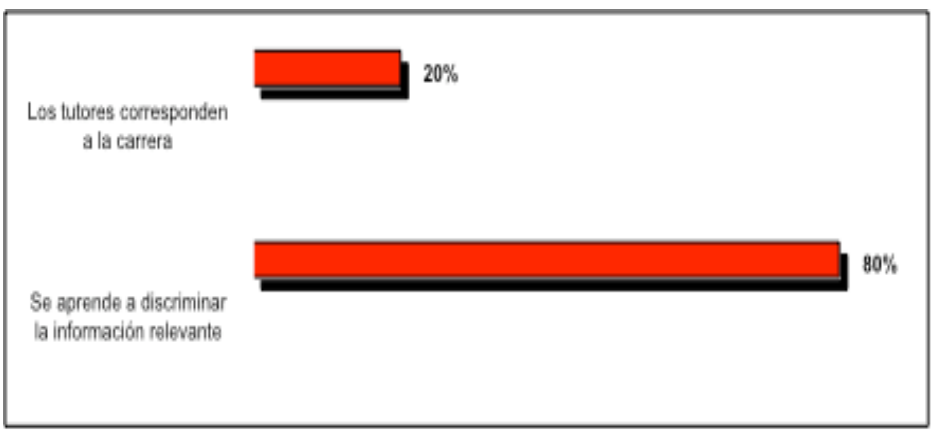

De acuerdo con los resultados entregados, y aunque parezca contradictorio con la pregunta anterior, hay alumnos que no creen haber vivido gradación de los aprendizajes en los diferentes módulos ABP, porque los problemas todos los años son muy parecidos. 


\subsection{Con respecto a los tutores con los cuales usted trabajó} ¿cómo cree que fue el trabajo de ellos? ¿Por qué?

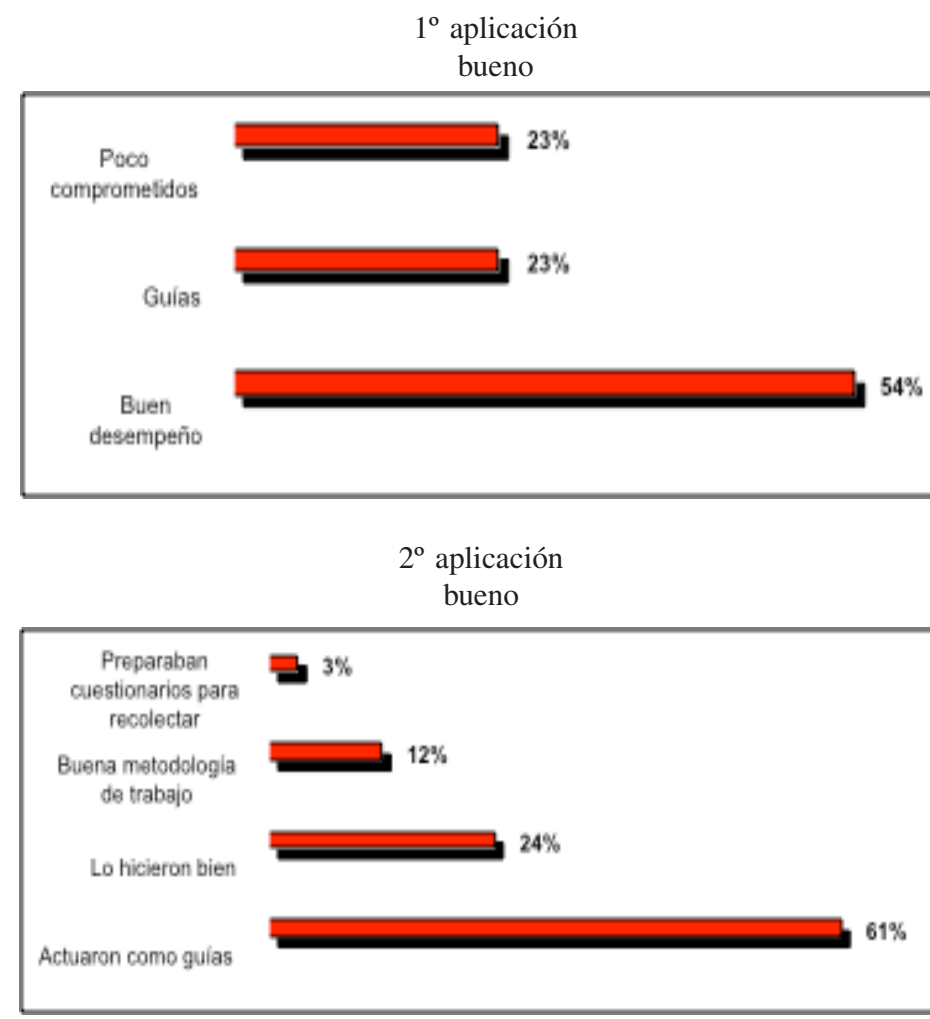

regular/malo

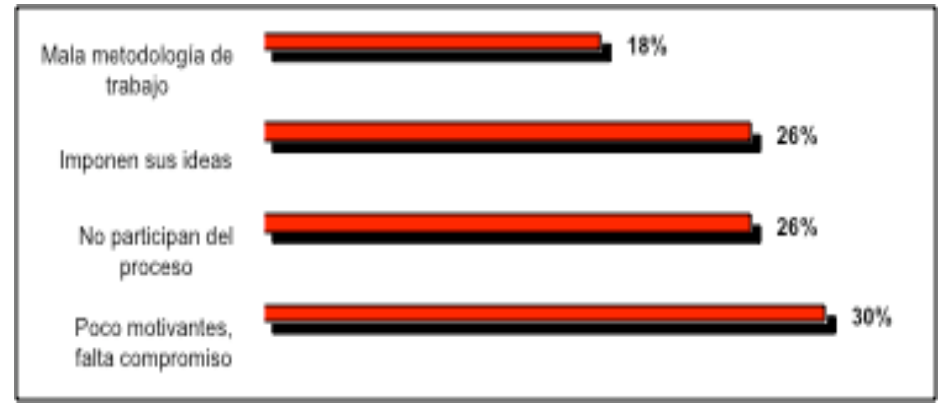

La gran mayoría de los alumnos consideró el aporte de sus diferentes tutores como bueno, pero no se puede desconocer que en la segunda aplicación de esta encuesta algunos alumnos no lo consideraron así. 


\subsection{Para usted ¿Cómo debería ser el tutor en los distintos niveles?}

$1^{\mathrm{o}}$ aplicación

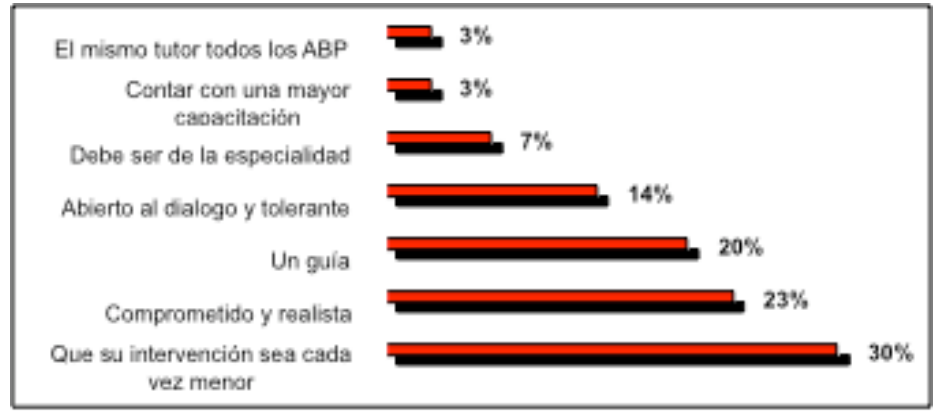

$2^{\circ}$ aplicación

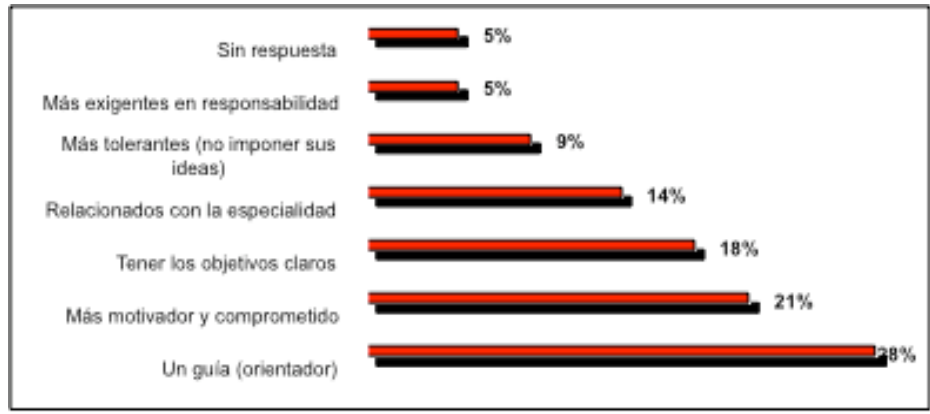

Los tutores, de acuerdo a los resultados obtenidos, deben ser un guía, pero su intervención debe ser cada vez menor en la medida que se avanza hacia los niveles superiores.

6.1. A la Comisión le interesa que ustedes den algunas sugerencias; a continuación le plantearemos algunos puntos. Si usted quiere dar sugerencias en otros aspectos lo puede hacer en el apartado OTROS.

$1^{\circ}$ aplicación

Organización de módulos

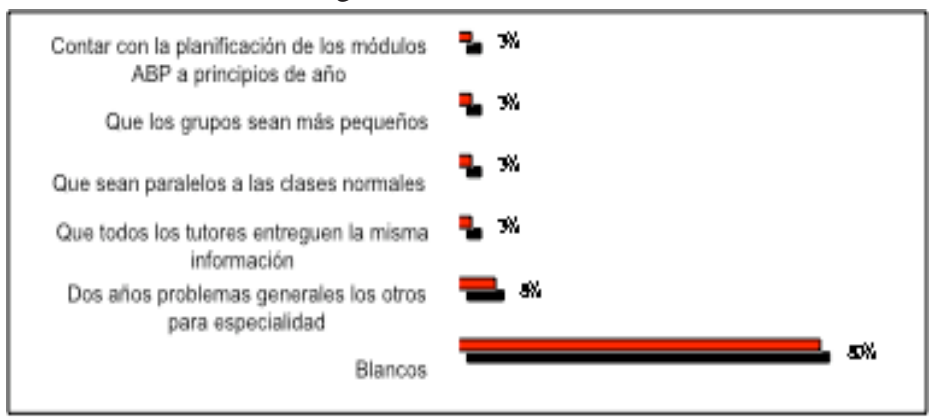


$2^{\circ}$ aplicación

Organización de módulos

Sin repuestas

Fechas

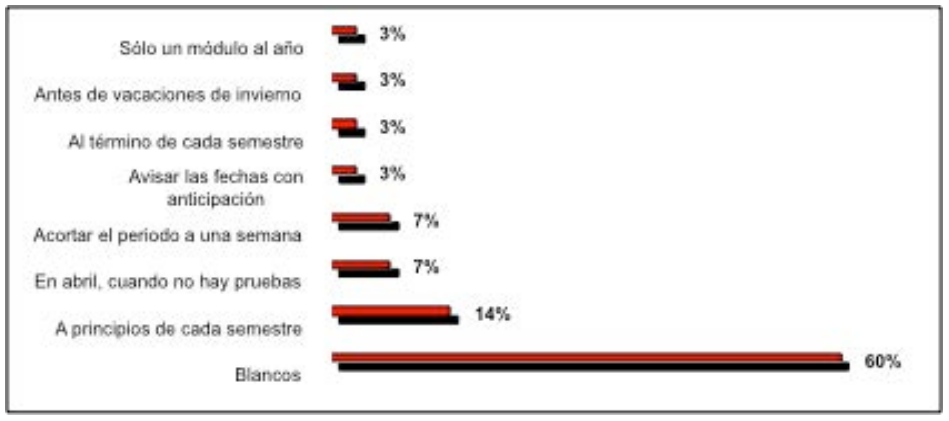

Fechas

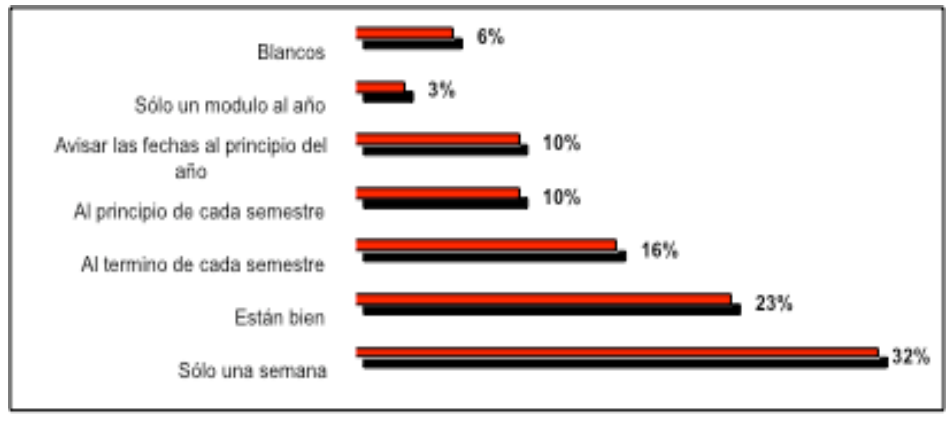

Tutores

\begin{tabular}{|rl|}
\hline Un mayor desempeño & $=3 \%$ \\
Que los tutores sean distintos en cada módulo & $=3 \%$ \\
Siempre el mismo tulió & $=3 \%$ \\
Que tengan mayor conocimianto del tema & $=3 \%$ \\
Más capacilación & $=7 \%$ \\
Que sean de la carrera & \\
Más nexibles & \\
Más compromiso & \\
Blancos & \\
\hline
\end{tabular}



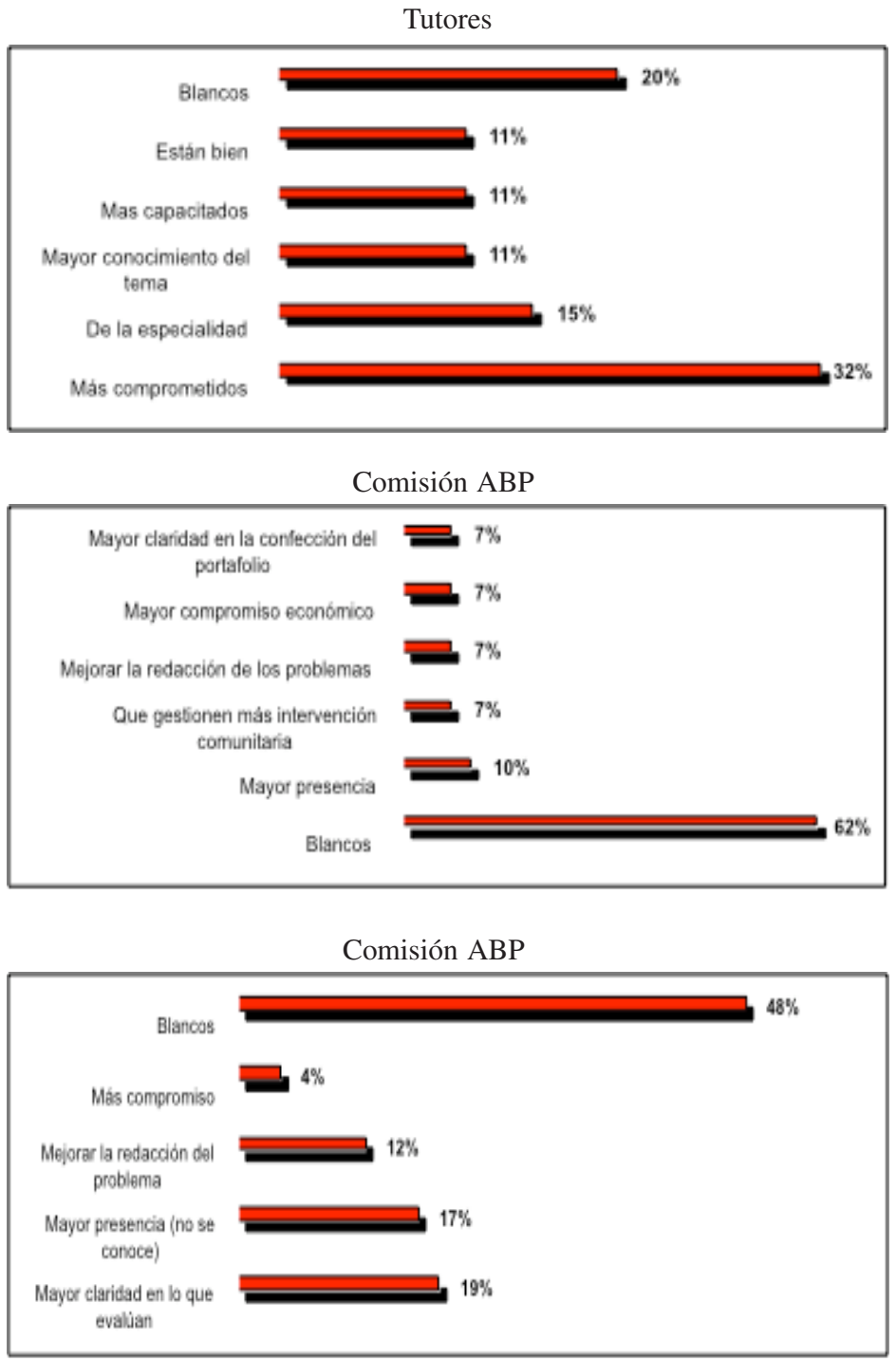

Problemas

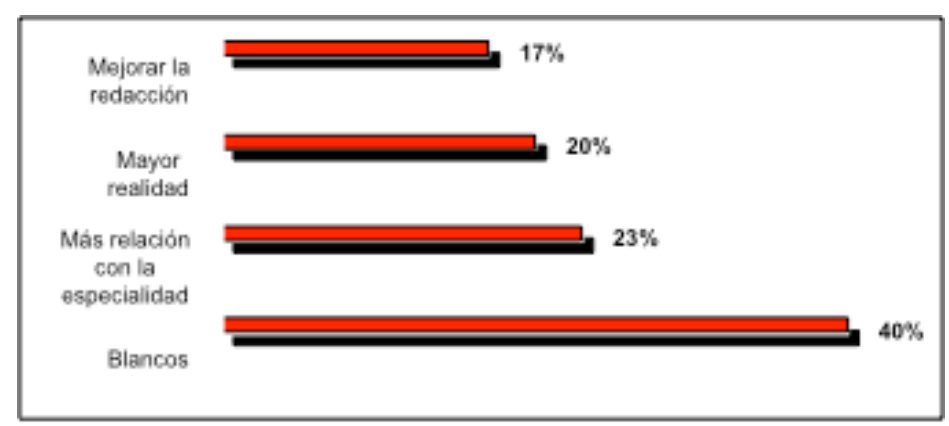


Problemas

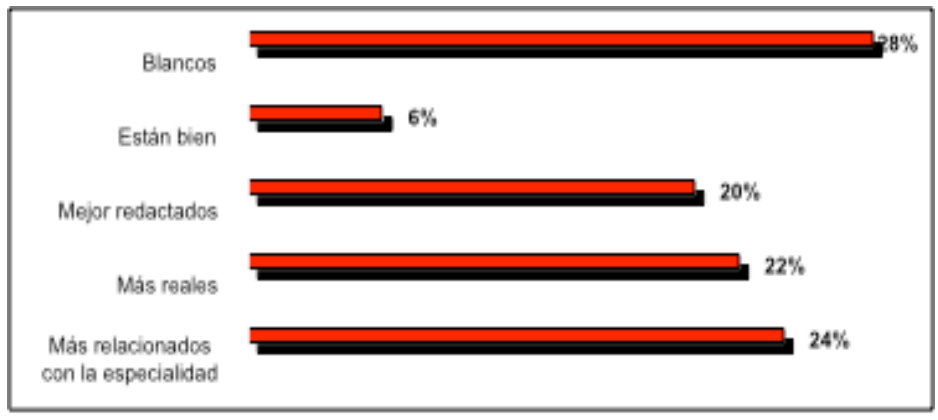

\section{Otros}

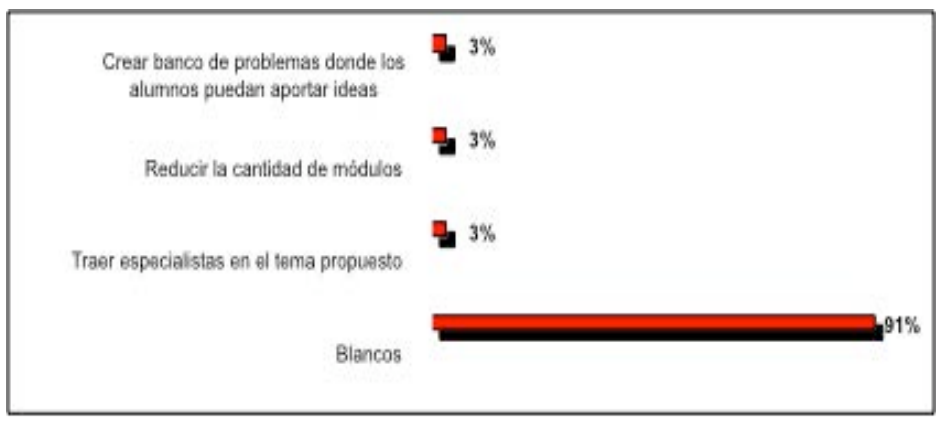

Otros

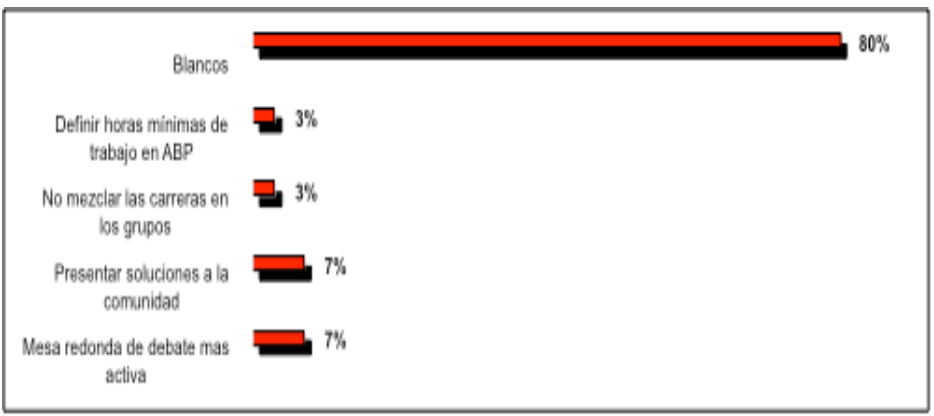

Realizar un análisis comparativo en esta pregunta es difícil porque los resultados entregados para las diferentes opciones, dentro de la pregunta, son muy variados, ya que algunos alumnos ni siquiera la consideraron como una alternativa de respuesta. 


\section{DISCUSIÓN DE LOS RESULTADOS}

\section{CUMPLIMIENTO DE OBJETIVOS}

En relación con el objetivo general, esta investigación nos ha permitido describir, de manera objetiva, la evaluación que cuatro cohortes de estudiantes de pedagogía hacen de la metodología $\mathrm{ABP}$, respecto de su formación profesional.

Desde la perspectiva de los objetivos específicos planteados por esta investigación, la información proporcionada por los alumnos nos permitió dar respuesta a tres aspectos importantes que necesitábamos evaluar:

1. Conocimiento del modelo ABP. Qué información manejan respecto de esta metodología de enseñanza-aprendizaje y qué efectos positivos perciben ellos, para su formación inicial docente, luego de haberse sometido a la experiencia ABP.

2. Determinar el cumplimiento de los objetivos de base del modelo y

3. Conocer la evaluación que ellos hacen respecto de la planificación, organización e implementación del sistema y su aplicación por parte de la Facultad.

Las respuestas recogidas en las dos aplicaciones de los años 2005 y 2007 nos permiten observar que:

En cuanto al conocimiento que los alumnos tienen respecto del ABP, ellos expresan que les permite un acercamiento a la realidad escolar, lo que consideran un valioso aporte para su formación. El abordar problemas extraídos de la realidad obliga al equipo a realizar investigación de campo. En este sentido, ellos valoran la experiencia de salir de la universidad para tomar contacto directo con profesores, directores de colegios, autoridades y profesionales que los orientan y ayudan a la construcción de sus soluciones.

En consecuencia, piensan que los principales objetivos del ABP son adquirir experiencia futura, desarrollar el trabajo en equipo y la investigación científica.

De alguna manera, esta aproximación concreta con la realidad educacional la valoran como una experiencia que les permite conocer anticipadamente el medio laboral (y sus problemas) en que se desempeñarán profesionalmente.

Para los estudiantes, el Aprendizaje Basado en Problemas les permite desarrollar cuatro principales habilidades a saber: (1) desarrollar una investigación basada en un método científico; (2) trabajar en equipo; (3) desarrollar pensamiento crítico y; (4) practicar la tolerancia.

Los encuestados responden, respecto a la gradación de los módulos, que a partir del nivel 100 (primer año) se plantea un Problema General; en el nivel 200 (segundo año), un tema más relacionado con la especialidad y en el nivel 300 (tercer año), problemas más específicos. También se aprecian opiniones de que los problemas abordan temáticas similares en el tiempo.

Los tutores son observados con un buen desempeño, no obstante, en algunas opiniones aparecen señalados con falta de compromiso. También los estudiantes observan que la intervención de los tutores debiera disminuir hacia los últimos niveles.

Las sugerencias en relación con la organización de los Módulos de ABP, en cuanto a fechas, tutores, comisión ABP, problemas y otros, en la mayoría de los casos han sido nulas. 


\section{CONCLUSIONES}

Sobre la base de los datos obtenidos, se puede concluir que el enfoque curricular de Aprendizaje Basado en Problemas es entendido por los estudiantes de Pedagogía de la Universidad de Atacama de la siguiente manera:

La metodología de Aprendizaje Basado en Problemas permite un acercamiento a la realidad escolar y sus objetivos consisten en entregar instancias en las cuales los estudiantes aprenden a trabajar en equipo, a desarrollar una investigación aplicando el método científico y a adquirir experiencias significativas en el ámbito de la docencia.

$\mathrm{Al}$ actuar como una herramienta que permite acercarse a la realidad escolar, el ABP es un gran aporte en la formación inicial docente, puesto que plantea problemas que ocurren en un contexto educativo y proporciona las bases para enfrentarse a diversas problemáticas.

El ABP es un proceso que, a lo largo de sus módulos, desarrolla principalmente cuatro habilidades: la primera habilidad consiste en que los estudiantes sean capaces de investigar aplicando el método científico; en segundo lugar, que los estudiantes logren trabajar en equipo; en tercer lugar fomenta el pensamiento crítico y, finalmente, incentiva la capacidad de tolerancia.

La gran mayoría de los estudiantes omitió su respuesta en relación a sugerencias para los aspectos operativos y de administración del ABP, sin embargo, es posible nombrar aquellas que, si bien no corresponden al mayor porcentaje, no dejan de ser importantes. Al respecto, los estudiantes indican que:

- los módulos de ABP deberían tener una duración de una semana.

- los tutores deberían ser más comprometidos.

- la comisión de ABP debería tener mayor presencia puesto que no se conoce.

- la comisión de ABP debería plantear con mayor claridad lo que evalúa.

- los problemas presentados deberían estar más relacionados con la especialidad.

Finalmente, estimamos importante la publicación de los resultados de esta investigación como una manera de informar a varias instituciones universitarias, del país y del extranjero, que están interesadas ${ }^{4}$ en implementar la metodología de Aprendizaje Basado en Problemas (ABP) en los currículos de formación de profesores, acerca de los logros y dificultades que hemos experimentado durante su aplicación en estos últimos ocho años. Y también motivar a otros centros de formación a vivir esta interesante y productiva experiencia pedagógica.

\section{REFERENCIAS BIBLIOGRÁFICAS}

Barrows, H.S. (1985). How to Design a Problem Based Learning Curricula For Preclinical Years. New York: Spring.

4 Nos han manifestado su interés y han conversado con nosotros al respecto, entre otras, las universidades de Tarapacá y la Cardenal Silva Henríquez, en Chile, y la Universidad Pedagógica de El Salvador y Universidad Univille, del Estado de Santa Catarina, Brasil, en el extranjero. 
Barrows, H. S. (1992). A Problem Based Learning in Secondary Education and PBL Institute. Springfield: Illinois.

Fosnot, C.T. (1996). Constructivism:Theory, Perspectivas, and Practice. New York: Teacher College Press.

Hargreaves, A.(2003). Enseñar en la Sociedad del Conocimiento. Barcelona: Octaedro.

Iglesias, J. (2002). El Aprendizaje Basado en Problemas en la Formación Inicial de Docentes. Perspectivas, Vol XXII, n. 3.

Magendzo, A. (1986). Currículo y Cultura en América Latina. Santiago: PIIE.

Marcelo, C. (1999). Formación del profesorado para el cambio educativo. Barcelona: EUB.

Proyecto "Docentes para el Siglo XXI: Reforma Integral del Currículo", 2000-UDA.

Savery ,J.R. y Duffy T. (1994). PBL An Instructional Model and Its Constructivist Framework. Educational Tehnology, vol. 35, n. 5, 31-38.

Schön, D.(1983). El profesional reflexivo. Como piensan los profesionales cuando actúan. Barcelona: Paidos.

Tardif, M. (2004). Los Saberes del Docente y su Desarrollo Profesional. Madrid: Nancea.

Comisión Aprendizaje Basado en Problemas. (2007). Aprendizaje Basado en Problemas: Una Nueva Propuesta Curricular en la Formación Inicial Docente de la Universidad de Atacama. Revista Foro Educacional, n. 11, 155-170. 
